\title{
Estudos sobre Envelhecimento no Brasil: Revisão Bibliográfica
}

\author{
StudesonAging inBrazil: LiteratureReview
}

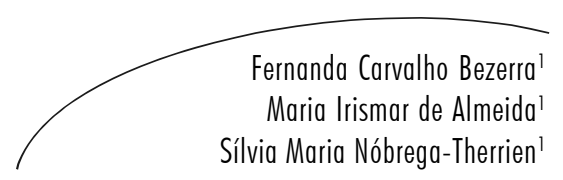

Resumo

O objetivo do presente trabalho foi realizar uma revisão bibliográfica dos estudos sobre envelhecimento no âmbito das ciências da saúde, a fim de apresentar um panorama do que tem sido pesquisado sobre o assunto na América Latina e no Brasil no período de 1982 a 2010. Utilizou-se a bases de dados LILACS disponível on line na Biblioteca Virtual em Saúde (BVS). Constatou-se um aumento expressivo do número de publicações a partir de 2006, principalmente nas categorias: estado de saúde $(51 \%, n=318)$, avaliação cognitiva $(12,72 \%, n=66)$, estudos sociodemográficos $(6,16 \% n=32)$, medicamentos $(3,27 \% n=17)$ e avaliação funcional $(3,08 \% \mathrm{n}=16)$. $\mathrm{Na}$ categoria estado de saúde, houve maior número de publicações relativas a atividade física $(17,9 \% \mathrm{n}=57)$, doenças cardiovasculares $(11,95 \% \mathrm{n}=38)$, dieta $(7,6 \% \mathrm{n}=24)$, saúde bucal $(6,6 \% \mathrm{n}=21)$, menopausa/ andropausa $(6,3 \% n=20)$, quedas $(5,97 \% n=19)$, atividade sexual $(5,03 \% n=16)$ e depressão $(4,1 \% \mathrm{n}=13)$. Concluiu-se que é importante estudar estes temas relacionados à senescência, para contribuir com a qualidade de vida nesta faixa etária.

\section{Abstract}

The main goal of this work is make a bibliographic revision of studies on aging in the field of health sciences, seeking to offer an outlook of the research on the subject carried out in Latin America and Brazil from 1982 to 2010. The LILACS online database available at the Virtual Health Library (BVS) was used for this purpose. It found a significant increase in the number of publications from 2006 on and a growing interest in the following categories: health condition $(51 \%$, $\mathrm{n}=318)$, cognitive evaluation $(12.7 \%, \mathrm{n}=66)$, socio-demographic studies $(6.1 \%$ $\mathrm{n}=32)$, medicines $(3.2 \% \mathrm{n}=17)$, and functional evaluation $(3.0 \% \mathrm{n}=16)$. Inside the health condition category, a high number of publications related to the

Palavras-chave:

Envelhecimento. Revisão bibliográfica. Ciências da Saúde.
Key words: Aging. Bibliographic revision. Health sciences.

\section{Correspondência}

Fernanda Carvalho Bezerra

Universidade Estadual do Ceará. Departamento de Saúde Pública

Av. Paranjana, 1700 - Campus do Itaperi

60740-000 - Fortaleza, CE, Brasil

Email: fernandacbezerra@hotmail.com 
following sub-categories was found: physical activity $(17.9 \% \mathrm{n}=57)$, cardiovascular deseases $(11.9 \% n=38)$, diet $(7.6 \% n=24)$, oral health $(6.6 \% n=21)$, menopause/ andropause $(6.3 \% n=20)$, falls $(5.9 \% n=19)$, sexual activity $(5 \% n=16)$ and depression $(4.1 \% \mathrm{n}=13)$. It was concluded that it also becomes important to study aspects related to aging, seeking to grant quality of life at this age stage.

\section{INTRODUÇÃO}

Estudos sobre as consequências do envelhecimento populacional nos países em desenvolvimento são bastante escassos e centramse, principalmente, nos processos relacionados às condições de saúde, como aposentadoria e arranjos familiares para o suporte dos idosos. ${ }^{1,2}$

O interesse em pesquisar o envelhecimento passou a ganhar maior importância recentemente nos países em desenvolvimento. Isso decorreu, sobretudo, do aumento acelerado da população acima de 60 anos em relação à população geral. ${ }^{2}$ O número de indivíduos com idade acima de 60 anos mais do que dobrou nos últimos 50 anos. ${ }^{2}$ Projeções recentes indicam que, em 2020, esse segmento poderá ser responsável por cerca de $14 \%$ $\left(n=30,9\right.$ milhões) da população brasileira. ${ }^{3}$

No Brasil, o número de idosos com idade acima de 60 anos passou de três milhões em 1960 para sete milhões em 1975, 14 milhões em $2002 \mathrm{e}$ 20 milhões (20.590.599) em 2010, um aumento de $600 \%$ em cinquenta anos e estima-se que em 2020 tal número alcançará 32 milhões. ${ }^{4} \mathrm{O}$ desafio maior no século XXI será cuidar dessa população crescente de idosos, a maioria com níveis socioeconômico e educacional baixos e elevada prevalência de doenças crônicas e incapacitantes. Os sistemas de saúde terão de fazer frente a uma crescente demanda por procedimentos diagnósticos e terapêuticos de doenças crônicas não-transmissíveis, principalmente as cardiovasculares e as neurodegenerativas, e a uma demanda ainda maior por serviços de reabilitação física e mental. ${ }^{4}$

Idosos, nas diferentes classes sociais, vivem a velhice de forma diversificada, como se o fim da vida reproduzisse e ampliasse as desigualdades sociais. $\mathrm{O}$ aumento do número de idosos em nosso país resulta da melhoria nas condições de saúde cujo reflexo é a maior sobrevivência da população. Embora isso represente um resultado positivo das ações governamentais, com o decorrer do tempo poderá constituir um problema de saúde. ${ }^{5} \mathrm{Com}$ efeito, é preciso, além da perspectiva adotada, que as ações dos profissionais da área da saúde e das ciências humanas sejam dirigidas à transformação dessa realidade, não apenas enfocando a velhice, mas também todas as fases da vida, nas suas diferentes abrangências - habitação, educação, saneamento, previdência, dentre outras.

O papel do idoso mostra-se diferente na sociedade atual, visto que a representação da velhice, como processo de perdas, foi objeto de uma inversão. ${ }^{6}$ Observa-se atualmente que essa etapa da vida é valorizada e privilegiada, tendo em vista as novas conquistas em busca de prazer, da satisfação e da realização pessoal, o que faz da Gerontologia objeto de crescente estudo.

O tema do envelhecimento da população brasileira só entrou realmente na agenda de pesquisa da Associação Nacional de Estudos Populacionais (ABEP) em 1988, durante o VI Encontro Nacional de Estudos Populacionais. ${ }^{7}$ A ABEP tem a finalidade de congregar estudiosos de Demografia para fomentar o intercâmbio científico na área, bem como ampliar e fortalecer o conhecimento da realidade demográfica nacional. Desde então, 51 trabalhos foram apresentados nos nove Encontros Nacionais e nove artigos foram publicados na Revista Brasileira de Estudos Populacionais, também da ABEP?

No Brasil, observa-se que o crescimento da população idosa desperta interesse para o desenvolvimento de pesquisas que abordem essa temática. Nota-se no cômputo dos trabalhos aqui 
mapeados, uma preocupação, ainda, com a necessidade de formação e capacitação de recursos humanos, fundamentada no cuidar gerontológico, para atender aos idosos.

Com base nestas considerações sobre o envelhecimento da população no Brasil, o objetivo desta pesquisa foi realizar uma revisão bibliográfica acerca de estudos do envelhecimento no âmbito das ciências da saúde. Acredita-se que a importância deste mapeamento na literatura está principalmente em poder fornecer um panorama sobre o que é pesquisado sobre o assunto no Brasil, evidenciar a realidade encontrada e elaborar algumas propostas de enfrentamento do desafio que o envelhecimento aporta para a atenção a saúde no Brasil.

\section{METODOLOGIA}

Foi realizada pesquisa bibliográfica descritiva, de caráter inventariante, com o objetivo de identificar produções científicas em periódicos nacionais e também em outros países da América Latina sobre o envelhecimento. Para isso, foi utilizado o banco de dados on line da Biblioteca Virtual em Saúde (BVS). ${ }^{8}$ Foram selecionados, ainda, os artigos na base de dados da Literatura Latino-Americana e do Caribe em Ciências da Saúde (LILACS), um índice bibliográfico relevante da literatura relativa às ciências da saúde que disponibiliza publicações, a partir de 1982, dos países da América Latina e Caribe. Assim, o mapeamento foi realizado com origem no acima referido ano.

A pesquisa foi realizada em janeiro de 2011, e utilizou-se a palavra-chave envelhecimento, tendo sido encontradas 3.193 publicações. Para trabalhar os dados, foi empregado um formulário avançado disponível em < http://bases.bvs.br/public/ scripts/php/page_show_main . $\mathrm{php}$ ?lang $=\mathrm{pt} \&$ form $=$ advanced $\&$ expression $>$.

Como critérios de inclusão, foram selecionadas publicações envolvendo seres humanos, na faixa etária acima de 60 anos e cuja palavra-chave foi envelhecimento. Quanto ao tipo de publicação, optou- se por artigos de revista, publicados no período de 1982 a 2010. Foram excluídas publicações que envolviam animais, faixa etária inferior a 60 anos e outros tipos de publicação, como ensaio clínico, relato de caso, publicações em livro ou congresso, por dificuldade de acesso às obras. Assim, o número de publicações encontradas, após refinamento dos dados, foi de 718 artigos, dos quais $519(\mathrm{n}=72,3 \%)$ abordavam assuntos relacionados aos tópicos do questionário do projeto SABE e $199(\mathrm{n}=27,7 \%)$ se referiam a outros assuntos. Dos 519 artigos selecionados, $318(\mathrm{n}=61,3 \%)$ se referiam ao tópico estado da saúde assunto abordado com maior frequência e 201 ( $n=38,7 \%)$ abordavam outros temas que contabilizavam nas categorias avaliação cognitiva $66(12,72 \%)$ publicações, estudos sociodemográficos $32(6,16 \%)$, medicamentos 17 (3,27\%) e avaliação funcional 16 (3,08\%).

Os resultados obtidos foram organizados por ano e por temática em gráficos e quadro. Para condensar os temas abordados na literatura sobre envelhecimento, utilizou-se como aporte de referência o questionário aplicado pelo Projeto Saúde, Bem-Estar e Envelhecimento (SABE), desenvolvido pela Organização Pan-Americana da Saúde (OPAS) com o objetivo de coletar informações sobre as condições de vida do idoso. Assim, surgiram os tópicos: avaliação cognitiva, estado de saúde, estado funcional, medicamentos, uso e acesso aos serviços de saúde, rede de apoio familiar e social, história de trabalho e fonte de receita, características da moradia, antropometria, mobilidade e flexibilidade, maus-tratos, avaliação da sobrecarga dos cuidadores, institucionalização, estudos epidemiossociodemográficos, estado da questão e lazer. Vale ressaltar que o tópico estado de saúde, no questionário do projeto $\mathrm{SABE}$, é organizado nos subtópicos hipertensão arterial sistêmica (HAS), diabetes mellitus (DM), doenças reumáticas, quedas, osteoporose, incontinência urinária e fecal, depressão, câncer, visão, audição, saúde bucal, prevenção de câncer de mama e de colo de útero, câncer de próstata, doenças sexualmente transmissíveis (DST), atividade sexual, hepatite, dieta, alcoolismo, tabagismo, atividade física, doenças pulmonares, cardiovasculares e acidente vascular encefálico (AVE). 
RESULTADOS

Os dados provenientes do mapeamento realizado foram organizados, portanto, após duas etapas de refinamento, quanto ao ano de publicação e temática em formato de gráficos e quadro, com a intenção de evidenciar um panorama de estudos sobre envelhecimento.
Ao quantificar a abordagem do tema envelhecimento, somente em revistas brasileiras, utilizando a base de dados LILACS, foram encontradas 350 publicações. Conforme mostra o gráfico 1 , observou-se que o número de publicações era relativamente pequeno e que passou a aumentar progressivamente a partir do ano 2000. Esse aumento foi mais expressivo no período compreendido entre os anos de 2006 e 2009.

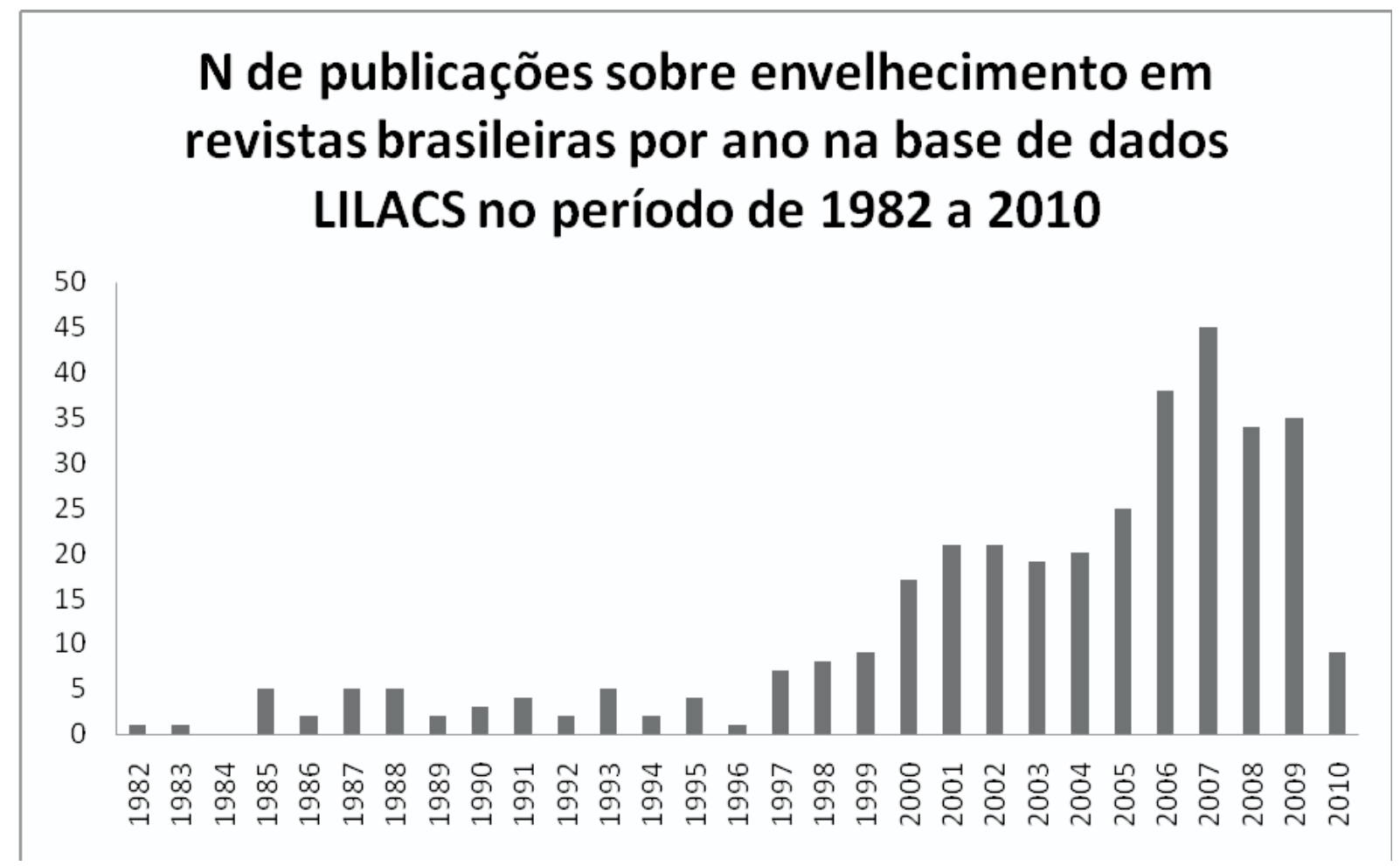

Gráfico 1 - Número de publicações por ano, citadas em revistas brasileiras, de 1982 a 2010, sobre o tema envelhecimento.

Quando se quantificou a abordagem do tema envelhecimento em revistas nacionais e internacionais na base de dados LILACS, foram encontradas 519 publicações. Conforme mostra o gráfico 2, observou-se aumento progressivo do número de publicações por ano, a partir de 1997, tendo sido o aumento mais expressivo no período compreendido entre 2006 e 2009. 


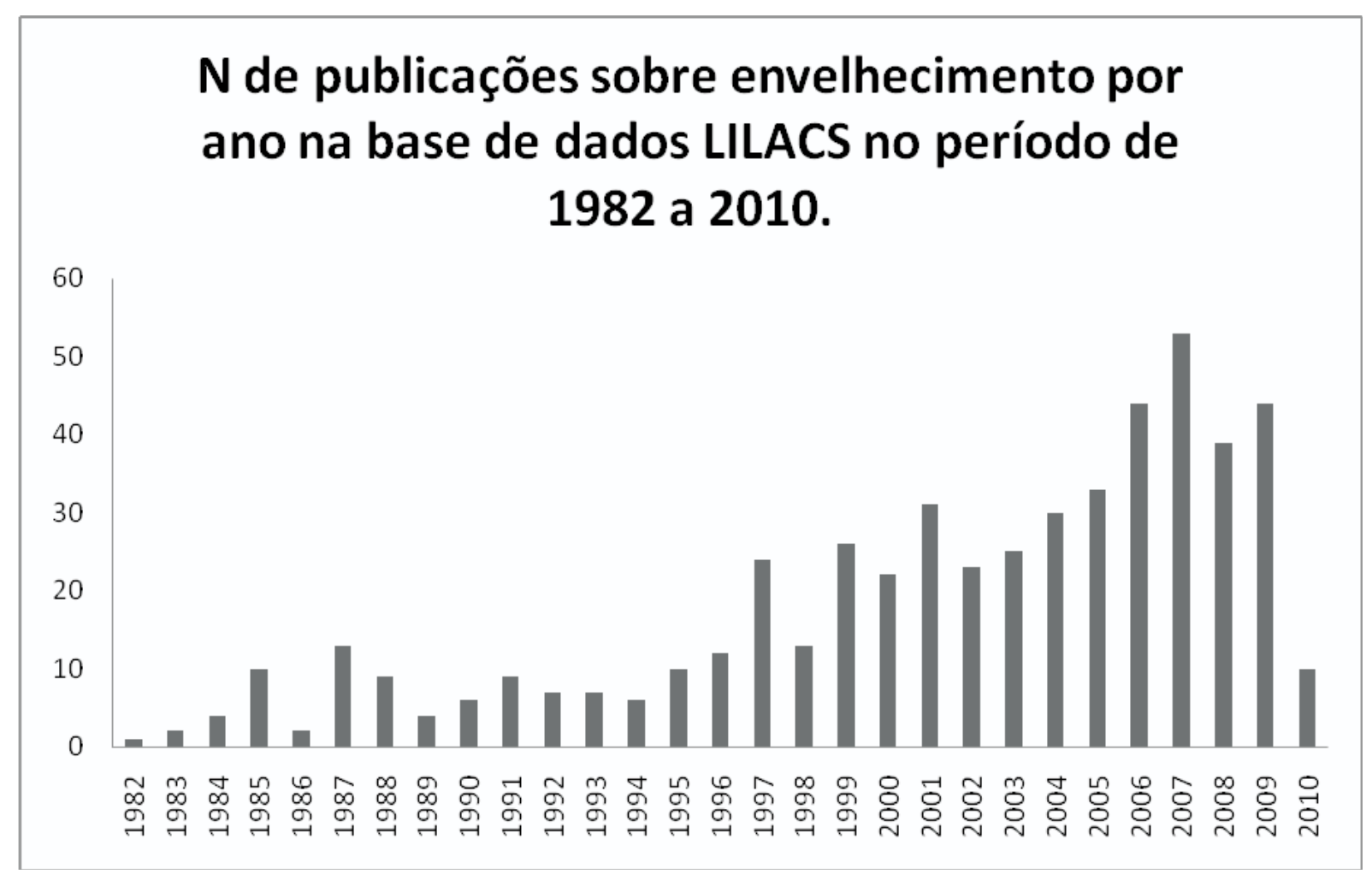

Gráfico 2 - Publicações por ano, citadas na base de dados LILACS, de 1982 a 2010, sobre o tema envelhecimento.

Conforme citado anteriormente, utilizou-se como referência o questionário SABE para enumerar os tópicos dentro da temática envelhecimento abordados em revistas nacionais e internacionais. Do total de 718 artigos encontrados, $519(\mathrm{n}=72,3 \%)$ publicações abordavam assuntos relacionados aos tópicos do questionário do projeto $\mathrm{SABE}$ e $199(\mathrm{n}=27,7 \%)$ publicações se referiam a outros assuntos. $\mathrm{O}$ tópico estado da saúde foi abordado com maior frequência, num total de 318 ( $\mathrm{n}=61,3 \%$ ) artigos, e decidiu-se analisá-lo separadamente.
Vale citar como são tratados outros tópicos relevantes dentro da área de ciências da saúde que contabilizaram 201 ( $\mathrm{n}=38,7 \%)$ publicações. Assim, avaliação cognitiva contabilizou $66(12,72 \%)$ publicações, estudos sociodemográficos $32(6,16 \%)$, medicamentos 17 (3,27\%) e avaliação funcional 16 (3,08\%), do total de 519 artigos selecionados.

Conforme mostra o gráfico 3, verificou-se maior interesse nas seguintes temáticas: estado de saúde (n $=51 \%)$, avaliação cognitiva $(\mathrm{n}=12,72 \%)$, estudos sóciodemográficos $\mathrm{n}=6,16 \%)$, medicamentos ( $\mathrm{n}$ $=3,27 \%)$ e estado funcional $(n=3,08 \%)$. 


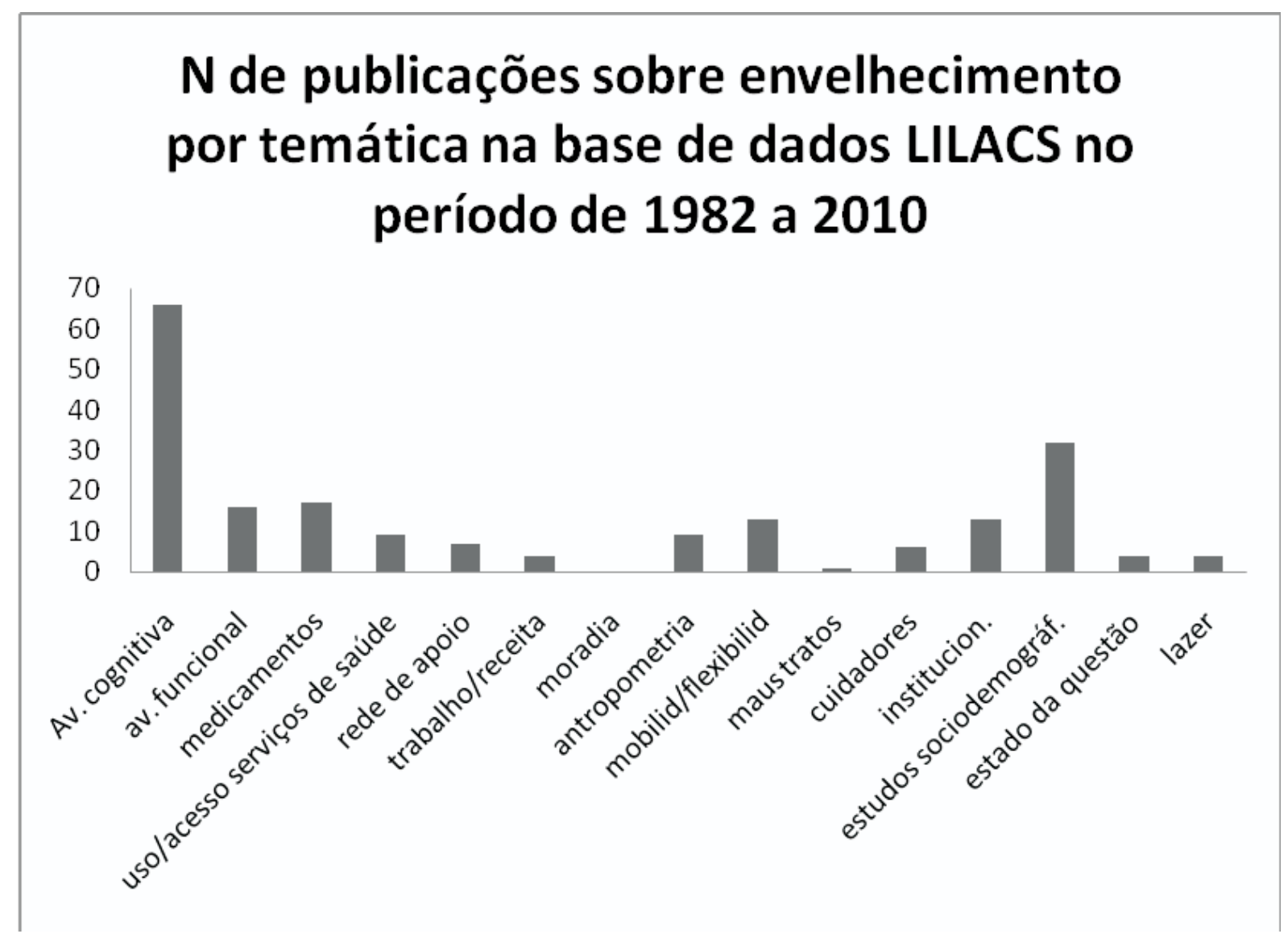

Gráfico 3 - Publicações por temática, citadas na base de dados LILACS, de 1982 a 2010, sobre o tema envelhecimento.

O tópico estado de saúde foi analisado separadamente, conforme pode ser observado no gráfico 4. Verificou-se maior interesse pelos subtópicos: atividade física $(17,9 \%, \mathrm{n}=57)$, doenças cardiovasculares $(11,95 \% \mathrm{n}=38)$ dieta $(7,6 \% \mathrm{n}=$ $24)$, saúde bucal $(6,6 \% \mathrm{n}=21)$, menopausa/ andropausa $(6,3 \% \mathrm{n}=20)$, quedas $(5,97 \% \mathrm{n}=19)$, atividade sexual $(5,03 \% \mathrm{n}=16)$ e depressão $(4,1 \%$ $\mathrm{n}=13)$. Observou-se também que $15,3 \%(\mathrm{n}=49)$ das publicações foram enquadradas como outros subtemas, pois não se relacionavam com as questões do tópico estado de saúde do projeto SABE. 


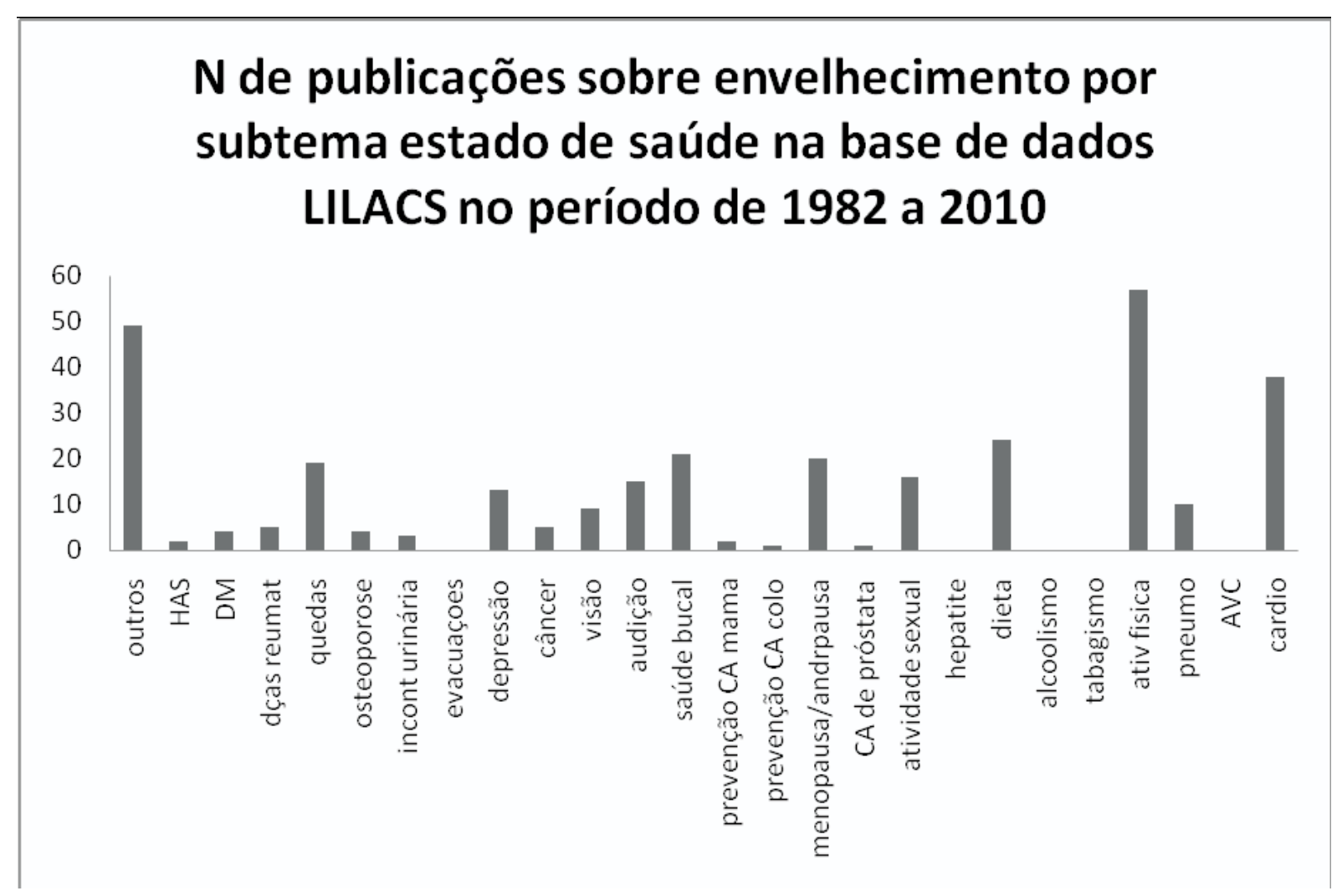

Gráfico 4 - Publicações por subtema estado de saúde, citadas na base de dados LILACS, de 1982 a 2010, sobre o tema envelhecimento.

Para avaliar se dentro das áreas de saúde houve mudança no interesse em estudar o envelhecimento, comparou-se a frequência com que o tema foi abordado no período de 1982 a 1999, em relação ao período de 2000 a 2010. De acordo com os dados apresentados no quadro 1, nota-se que houve aumento do número de publicações em praticamente todas as áreas de saúde, exceto na Fisiologia, em que se detectou um decréscimo de $3,08 \%(\mathrm{n}=19)$. Vale ressaltar que, na área de Educação Física, o aumento do número de publicações foi mais marcante: $8,67 \%$. $(n=45)$. 
Quadro 1 - Comparação do número de publicações, por área de saúde, nos períodos de 1982-1999 e 2000-2010.

\begin{tabular}{|lccc|}
\hline \multicolumn{1}{|c|}{ Período } & & & \\
& $\mathbf{1 9 8 2} \mathbf{- 1 9 9 9}$ & $\mathbf{2 0 0 0} \mathbf{- 2 0 1 0}$ & $\mathbf{\%}$ \\
\hline Fórea & & & 3,08 \\
Educação física & 0 & 47 & 8,67 \\
Odontologia & 2 & 16 & 1,73 \\
Enfermagem & 7 & 30 & 4,82 \\
Medicina & 5 & 119 & 5,39 \\
Fisioterapia & 91 & 20 & 3,66 \\
Psicologia & 1 & 33 & 4,24 \\
Gerontologia & 11 & 15 & 1,93 \\
Nutrição & 5 & 17 & 1,73 \\
Fisiologia & 8 & 6 & $-3,08$ \\
Farmacologia & 22 & 4 & 0,77 \\
Saúde Pública & 0 & 33 & 4,24 \\
\hline Total & 11 & 356 & 37,18 \\
\hline
\end{tabular}

\section{DISCUSSÃO}

Ao avaliar o número de publicações por ano sobre o tema envelhecimento, verificou-se que, tanto no Brasil como nos demais países da América Latina, houve aumento do número de artigos a partir de 2000 , tendo sido mais expressivo entre 2006 e 2009 (gráficos 1 e 2). O interesse em estudar o envelhecimento populacional poderia ser explicado pela transição demográfica experimentada pelos países em desenvolvimento. Houve aumento considerável da população acima de 60 anos de idade no Brasil ${ }^{4}$ e são esperados aumentos de até $300 \%$ na população idosa nos países da América Latina nos próximos 30 anos. ${ }^{9}$ Outro fator é a aprovação do Estatuto do Idoso no Brasil, em setembro de 2003, e sua repercussão entre os países vizinhos. Além desses fatores, a criação de faculdades e cursos de pós-graduação, que impulsionaram o estudo e a produção dos grupos e das linhas de pesquisa na área, bem como a implementação das especializações em Geriatria e Gerontologia na área de saúde do idoso, também pode ter contribuído para despertar o interesse em estudar o tema ora focalizado.
Para fins de comparação, cita-se o trabalho de Santos-Filho et al., ${ }^{10}$ que realizaram revisão de literatura com o intuito de analisar o número de publicações científicas por ano sobre o tema do envelhecimento, no período compreendido entre 1949 e 2004, em indexadores como o Pubmed, um banco de dados que possibilita a pesquisa bibliográfica em mais de 17 milhões de referências de artigos médicos. Ao contrário do observado neste estudo, em que se detectou aumento do número de publicações a partir de 2000 , SantosFilho et al. ${ }^{10}$ verificaram um crescente interesse em estudar o envelhecimento mais precocemente a partir de 1986. Uma justificativa para tal disparidade de resultados poderia decorrer do fato de a transição demográfica nos países desenvolvidos ter ocorrido mais cedo do que na América Latina.

Quando se analisou o número de publicações por temática em revistas nacionais e internacionais, verificou-se maior interesse nos seguintes tópicos: estado de saúde, avaliação cognitiva, estudos sóciodemográficos, medicamentos e estado funcional (gráfico 3). Notou-se número expressivo de artigos que 
abordavam o estado de saúde. Assim, decidiu-se dividi-lo em subtópicos (gráfico 4) e constatou-se que os subtemas atividade física, doenças cardiovasculares, dieta, saúde bucal, menopausa/ andropausa, quedas, atividade sexual e depressão eram abordados com maior frequência.

A idade é um fator de risco para cardiopatias. Assim, com o aumento da expectativa de vida, torna-se necessário estudar as doenças cardiovasculares. Foram encontrados diversos artigos de revisão com objetivo de compartilhar e difundir conhecimentos a respeito das particularidades da propedêutica cardiovascular no idoso. Escobar ${ }^{11}$ relata que as cardiopatias mais frequentes entre os idosos são doença coronariana, hipertensão arterial e as valvopatias. Escobar ${ }^{11}$ também chama atenção para o fato de que o uso de fármacos deve ser cuidadoso e iniciado com doses menores do que as habituais. Cunha et al. ${ }^{12}$ avaliaram a frequência da prescrição de digital em idosos admitidos a unidade de Geriatria de um hospital geral e detectaram elevada prevalência de prescrição deste medicamento nos idosos admitidos e, quando avaliaram a indicação da prescrição, observaram razões consideradas inadequadas ou questionáveis. Em decorrência do maior risco de intoxicação digitálica nessa faixa etária, a droga deveria ser prescrita sob indicações mais criteriosas.

Em outro estudo, Duarte et al. ${ }^{13}$ avaliaram em pacientes submetidos a cintiografia de reperfusão do miocárdio (CPM) o grau de associação entre fatores de risco cardiovascular e a presença de doença arterial coronária (DAC). Envelhecimento e sexo masculino foram os principais fatores de risco encontrados para DAC. Diabetes mellitus (DM) e dislipidemia apresentaram maior associação com a presença de DAC no homem e o DM na mulher, sendo tais fatores de risco passíveis de controle por meio da dieta.

Loures et al. ${ }^{14}$ avaliaram, retrospectivamente, 75 pacientes com idade entre três e 70 anos submetidos a operação cardíaca no HC-UFPR, entre 1995 e 1999, com o objetivo de analisar os resultados pós-operatórios imediatos e tardios. Concluíram que, apesar de serem pacientes de maior complexidade clínica pela maior incidência de doenças crônicas e acometimento de outros órgãos, os avanços na cirurgia cardíaca e terapia intensiva tornaram possível a intervenção com baixa morbimortalidade.

Com relação ao tema dieta, o interesse crescente em abordá-lo relaciona-se ao fato de que o estado nutricional é importante marcador de saúde no idoso, e que, durante o envelhecimento, ocorrem alterações fisiológicas que podem influenciar a nutrição. Diversos fatores podem contribuir para o desenvolvimento de distúrbios nutricionais, como limitações financeiras, incapacidade física para o preparo dos alimentos, distúrbios mentais e isolamento social. ${ }^{15-17} \mathrm{~A}$ presença de enfermidades e o uso de medicamentos que afetem a sensibilidade gustativa também podem interferir na aceitação da dieta pelo idoso. ${ }^{16}$ Por outro lado, a obesidade tem prevalência aumentada tanto em jovens como em idosos. ${ }^{18}$ Por ser fator de risco para HAS, DM não insulino-dependente e doenças cardiovasculares, enfermidades com prevalências já elevadas entre pessoas idosas, a obesidade deve ser considerada um problema de saúde pública.

O envelhecimento pode também predispor a alterações fisiológicas e patológicas na dentição e na articulação temporomandibular (ATM), bem como modificações sistêmicas. Assim, o aumento da expectativa de vida é acompanhado por necessidades odontológicas cada vez mais complexas e em maior quantidade. No âmbito da saúde bucal, observou-se interesse em abordar a influência dessas particularidades do idoso na escolha da conduta mais apropriada e na resposta ao tratamento. Dessa forma, Rivaldo et al. ${ }^{19}$ realizaram uma revisão sobre alterações fisiológicas e patológicas relacionadas com a saúde e as condições bucais dos idosos, de maneira a contribuir para a disseminação desta informação entre os profissionais da Odontologia.

Umbelino et al. ${ }^{20}$ avaliaram 512 pacientes idosos atendidos na Disciplina Estomatologia II, da Faculdade de Odontologia da Universidade do Estado do Rio de Janeiro (FO/UERJ), e observaram que a reabsorção do rebordo alveolar 
e varicosidades linguais foram alterações de desenvolvimento prevalentes. Com relação às alterações patológicas, a língua saburrosa caracterizou a lesão dominante em ambos os gêneros, seguida pela hiperplasia fibrosa inflamatória e candidíase atrófica. Azcona ${ }^{21}$ ressaltou a importância de o odontólogo estudar as características da deterioração da ATM antes de escolher o tratamento, porque algumas vezes não é fácil distinguir um processo involutivo próprio da idade de uma enfermidade.

A respeito dos temas menopausa/andropausa, observou-se que este é abordado com maior frequência por especialistas em Ginecologia e Endocrinologia. Santos \& Jeckel-Neto ${ }^{22}$ relatam que, com o aumento da expectativa de vida em ambos os sexos, surgiu o interesse em estudar o descenso genital numa perspectiva de propiciar melhor qualidade de vida para o idoso, uma vez que tal condição não diminui a longevidade, mas pode limitar o convívio social e o desempenho das atividades diárias.

Moreira $^{23}$ elaborou uma revisão sobre as alterações na estrutura do músculo estriado da mulher durante o climatério e ressaltou a importância de discutir as causas e consequências da perda de volume de músculo, conhecida como sarcopenia, pois suas consequências alteram substancialmente a qualidade de vida. Yabur ${ }^{24}$ discutiu doenças crônicas degenerativas relacionadas com os níveis de estrógeno, tais como doenças cardiovasculares e doença de Alzheimer; bem como os efeitos da terapia de reposição hormonal (TRH) na redução de fraturas por osteoporose e no aumento do risco de câncer de mama e de endométrio.

O declínio progressivo da produçäo androgênica pode ser encontrado em pelo menos $20 \%$ dos homens com idade entre 60 e 70 anos, e algumas vezes se inicia a partir dos 50 anos. ${ }^{25,26}$ Bonaccorsi ${ }^{25}$ sugere, entretanto, que a TRH deve somente ser considerada em presença de níveis séricos de testosterona abaixo dos limites normais mínimos para adultos jovens, acompanhada de sinais inequívocos de insuficiência androgênica, e que é necessário, antes de iniciar a TRH, excluir outras causas reversíveis de hipoandrogenismo e contraindicaçöes ao tratamento.

O tema atividade sexual foi abordado com relativa frequência. Bohórquez $\&$ Julián ${ }^{27}$ discutiram a negação, por parte da população, inclusive por profissionais de saúde, da sexualidade na senilidade. A atividade sexual nos idosos tem características particulares que obedecem às mudanças anatômicas e fisiológicas do envelhecimento. Além disso, condições como a polifarmácia e a multipatologia podem afetar de maneiras diversas a função sexual. Assim, é necessário que médicos e outros profissionais intervenham, possibilitando o diagnóstico de disfunções, a terapia farmacológica e a desmistificação do tema. Em outro estudo, Polizer $\&$ Alves $^{28}$ avaliaram o perfil de satisfação e função sexual de mulheres idosas e detectaram que a maioria das entrevistadas teve padrão de desempenho/satisfação regular a bom, sem grandes alterações da função sexual. Também relataram que as alterações da resposta sexual consideradas fisiológicas nesta fase da vida não determinam o fim da vida sexual das mulheres idosas entrevistadas.

Vale ressaltar a importância de trabalhos a respeito da presença de Aids/HIV na terceira idade. Assim, Jailson \& Silva ${ }^{29}$ observaram aumento da incidência de Aids em indivíduos acima de 50 anos, em 1998, após a introdução de medicamentos para disfunção erétil. Vasconcelos et $a 1 .{ }^{30}$ estudaram o perfil epidemiológico de pacientes com HIV/Aids no Hospital Correia Picanço (Recife-PE) e detectaram aumento no número de casos em mulheres, principalmente as casadas, em heterossexuais e predomínio em indivíduos de baixa escolaridade. Em razão de tais resultados, sugeriram a necessidade $d a$ elaboraçäo de trabalhos educativos de maneira a atingir os maiores de 60 anos.

Diversos profissionais da área de saúde, como médicos, fisioterapeutas e fonoaudiólogos, estudam o tema quedas. Os artigos que abordavam este tema tinham como objetivo reunir os fatores que contribuiam para a maior prevalência de quedas nos idosos em comparação com a 
população mais jovem e apresentar opções para a prevenção. Dessa forma, Guimarães \& Farinatti ${ }^{31}$ observaram que a deterioração da visão, uso simultâneo de medicamentos (especialmente diuréticos e psicoativos) e flexibilidade reduzida (quadril e tornozelos) parecem associar-se com a frequência de quedas. Ishizuka et al..$^{32}$ relataram depressão e fraqueza muscular como fatores relacionados ao risco de queda. Cartier $^{33}$ relata uma relação entre alteração da marcha no idoso e maior frequência de queda. Assim, a menor amplitude de movimento da pelve e doenças que alteram o centro de equilíbrio do corpo como doença de Parkinson, paraparesias espásticas, AVE ou neuropatias podem prejudicar a marcha.

No tema depressão, alguns artigos sugeriam que a prática de exercícios físicos, bem como a participação em grupos de educação permanente, como as universidades para a terceira idade, poderiam ser fatores de melhora da depressão. Assim, Irigaray et al. ${ }^{34}$ apontaram para a existência de uma associação entre menor intensidade de sintomatologia depressiva e tempo de participação na UNITI (Universidade para a Terceira Idade) superior a um ano. Moraes et al. ${ }^{35}$ realizaram revisão de literatura para investigar o papel protetor do exercício físico e sua eficácia no tratamento da depressão. Concluíram que a depressão promove redução da prática de atividades físicas e que a atividade física pode ser um coadjuvante na prevenção e no tratamento da depressão no idoso.

Quando se avaliou, dentro das áreas de saúde, se houve mudança ao longo do tempo no interesse em estudar o envelhecimento (quadro 1), notouse aumento do número de publicações em praticamente todas as áreas de saúde, exceto na de Fisiologia, como já se adiantou, em que se detectou decréscimo de 3,08\% $(\mathrm{n}=19)$. Tal redução pode ser resultado da ampliação dos cursos de graduação e pós-graduação nas diversas especialidades. Assim, os assuntos podem ter sido canalizados para outras áreas de saúde.

Observação importante foi o aumento do número de publicações mais expressivo na área de Educação Física. Tal fenômeno pode decorrer do consenso atual acerca da importância de cultivar estilos de vida mais saudáveis para prevenir doenças e reduzir custos para o sistema de saúde. Detectou-se o fato de que não apenas estudiosos do curso de Educação Física, mas também médicos, psicólogos e fisioterapeutas, se interessam por comprovar o efeito benéfico da prática contínua de exercìcios por idosos. Em trabalho realizado por Garrett et al., ${ }^{36}$ foram avaliados os custos do sedentarismo entre os membros de um plano de saúde do Estado de Minesota, nos Estados Unidos, tendo-se constatado que a doença cardíaca representou a maior despesa para o plano de saúde, custando 35,3 milhões de dólares em 2000.

\section{CONSIDERAÇÕES FINAIS}

Este ensaio revela a ausência de trabalhos sobre alguns temas relevantes, no período 1982 a 2010 e na base de dados pesquisada, tais como tabagismo, alcoolismo, hepatite e AVE. Também foram escassas publicações sobre câncer de mama, colo de útero e próstata. Por outro lado, um maior número de trabalhos está situado na temática de estado de saúde, mais precisamente nos subtemas atividade física e doenças cardiovasculares. É importante que se realizem estudos tanto nos temas em que existem poucas publicações como naqueles que já são abordados com certa frequência.

Com o aumento da expectativa de vida, os processos relacionados ao envelhecimento precisam ser estudados pelas diversas áreas de saúde, com um enfoque interdisciplinar, a fim de possibilitar a promoção de saúde e prevenção de doenças.

A base de dados LILACS contempla publicações pertinentes a 27 países, a partir de 1982, o que representa limitações ao estudo. Assim, não foi possível detectar o panorama dos estudos sobre envelhecimento antes do ano supracitado e em países europeus e da América do Norte. 


\section{REFERÊNCIAS}

1. Moreira MM. Envelhecimento da população brasileira. Belo Horizonte. Tese [Doutorado em Saúde]. -Universidade Federal de Minas Gerais; 1997.

2. Dias Júnior CS, Costa CS, Lacerda MA. O envelhecimento da população brasileira: uma análise de conteúdo das páginas da REBEP. Rev. Bras. Geriatr. Gerontol 2006; 9 (2).

3. Beltrão KI, Camarano AA , Kanso S. Dinâmica populacional brasileira na virada do século XX. Rio de Janeiro: Ipea; 2004.

4. Lima-Costa MF, Veras R. Saúde pública e envelhecimento. Cad. Saúde Pública 2003; 19: 700-701.

5. Haddad EGM. A velhice em movimento. Gerontol 1993; 1(1)29-30.

6. Debert GG. Antropologia e o estudo dos grupos e das categorias. In: Moraes M, Barros L. Velhice ou terceira idade? Rio de Janeiro: Fundação Getúlio Vargas; 1998. p.49-67.

7. Camarano AA. Envelhecimento da População Brasileira: uma contribuição demográfica. In: Freitas EV . Tratado de Geriatria e Gerontologia 2. ed. Rio de Janeiro: Guanabara Koogan; 2006. p 88.

8. Biblioteca Virtual em Saúde. Literatura científica e técnica. São Paulo: BVS; 2009. Disponível em: http://www.bireme.br/php/ index.php.

9. Truelsen T, Bonita R, Jamrozik K. Survillance of stroke: a global perspective. Int J Epidemiol 2001; 30(S11-S12).

10. Santos-Filho SD ,et al. O interesse científico no estudo do envelhecimento e prevenção em ciências biomédicas. Rev Bras Ciênc Envelhec Humano $2006 \mathrm{jul} / \mathrm{dez}$; 9(2): 70-78

11. Escobar CE. Cardiología geriátrica, envejecimiento y aparato cardiovascular Rev. sanid. def. nac. 1994; 11(2):135-40

12. Cunha UG, et al. Uso de digital em idosos admitidos em unidade de geriatria de hospital geral Arq. bras. Cardiol 1998 nov; 71(5):695-8.

13. Duarte P S, et al. Associação entre fatores de risco para doença arterial coronariana e coronariopatia em pacientes submetidos a cintilografia de perfusão do miocárdio Arq. bras. Cardiol 2007 mar ;88(3):304-313.

14. Loures DR, et al. Cirurgia cardíaca no idoso: Rev. bras. cir. Cardiovasc 2000 jan/mar; 15(1):1-5.
15. Campos MAG, et al. Estado nutricional e antropometria em idoso: revisão da literatura. Rev. méd. Minas Gerais 2007 jul/dez ;17(3/ 4):111-120.

16. Monteiro MAM. Percepção sensorial dos alimentos em idosos. Espaç. saúde 2009 jun;10(2):34-42.

17. Sampaio LR. Avaliação nutricional e envelhecimento. Rev. nutr 2004 out/dez; 17(4):507-514.

18. Marques APO, et al. Envelhecimento, obesidade e consumo alimentar em idosos Rev. bras. geriatr. gerontol 2007 ago;10(2):231-242.

19. Rivaldo EG, et al. Envelhecimento e saúde bucal Stomatos 2008 jan/jun;14(26):39-45.

20. Umbelino Júnior AA, Apolinário $\mathrm{T}$ de $\mathrm{O}$, Cantisano MH. Condições bucais e sistêmicas de um grupo de pacientes da terceira idade atendidos na FO/UERJ Rev. bras. Odontol 2009 jul/dez ;66(2):183-186.

21. Azcona S. Envejecimiento articular Claves odontol 2004 jul; 11(56):9-12.

22. Santos TG, Jeckel-Neto EA. Envelhecimento e descenso genital: o papel do colágeno. Rev Femina 2004 out; 32(9):741-9.

23. Moreira MA. Climatério e sarcopenia: o pouco que se sabe não é valorizado Femina 2008 abr; 33(4):261-265.

24. Yabur JA. La menopausia puesta al día Gac. méd. Caracas 2006 mar; 114(1):1-12.

25. Bonaccorsi AC. Andropausa: insuficiência androgênica parcial do homem idoso: uma revisäo Arq. bras. endocrinol. Metab 2001 abr; 45(2):123-133.

26. Devoto CE, Aravena CL. Hipogonadismo associado a la senilidad en el varon (climaterio masculino - andropausia - Adam) Rev. chil. obstet. Ginecol 2004 ;69(5):392-398.

27. Bohórquez C, Julián D. Sexualidad y senectud Hacia promoc. Salud 2008 ene/dic; 13:13-24.

28. Polizer AA, Alves TMB. Perfil da satisfação e função sexual de mulheres idosas Fisioter. mov $2009 \mathrm{abr} /$ jun ;22(2):151-158.

29. Jailson L, Silva MDP. Tendência de AIDS no grupo etário de 50 anos e mais no período anterior e posterior à introdução de medicamentos para disfunção erétil: Brasil ,1990 a 2003 Rev. bras. geriatr. Gerontol 2007 ago;10(2):203-216. 
30. Vasconcelos EMR, Alves FAP, Moura LML . Perfil epidemiológico dos clientes HIV/AIDS na terceira idade Rev. bras. Enferm 2001; 54(3):435-445.

31. Guimarães JMN, Farinatti PTV. Análise descritiva de variáveis teoricamente associadas ao risco de quedas em mulheres Rev. bras. med. Esporte 2005 set/out; 11(5):299-305.

32. Ishizuka MA, et al. Falls by elders with moderate levels of movement functionality. Clinics 2005 jan/fev; 60(1):41-46.
33. Cartier RL. Caídas y alteraciones de la marcha en los adultos mayores. Rev. méd. Chile 2002;130(3):332-337.

34. Irigaray TQ, Schneider RH. Prevalência de depressão em idosas participantes da universidade para a terceira Idade Rev. Psiquiatr 2007 jan/jun ;29(1):19-27.

35. Moraes $\mathrm{H}$, et al. $\mathrm{O}$ exercício físico no tratamento da depressão em idosos: revisão sistemática. Rev. psiquiatr 2007 jan/abr ; 29(1):70-79.

36. Garrett NA, et al. Physical inactivity: direct cost to a health plan. Am J Prev Med 2004; 27:304-9. 
